\title{
Retinal vessel calibre and micro- and macrovascular complications in type 1 diabetes
}

\author{
J. Grauslund • L. Hodgson • R. Kawasaki • A. Green • \\ A. K. Sjølie • T. Y. Wong
}

Received: 22 April 2009 /Accepted: 22 June 2009 / Published online: 18 July 2009

(C) Springer-Verlag 2009

\begin{abstract}
Aims/hypothesis The purpose of the study was to evaluate the association between retinal vascular calibre and microand macrovascular complications in a population-based cohort of Danish type 1 diabetic patients.

Methods This was a cross-sectional study of 208 longsurviving type 1 diabetic patients from a population-based Danish cohort. Retinal photographs were obtained at a clinical examination attended by each participant in 20072008, and retinal vascular calibre was measured and summarised as the central retinal artery or vein equivalent (CRAE or CRVE) using a computer-based program and a standardised protocol. Associations between retinal vascular calibre and micro- and macrovascular complications were examined after adjusting for confounding clinical characteristics.
\end{abstract}

J. Grauslund $(\bowtie) \cdot$ A. K. Sjølie

Department of Ophthalmology, Odense University Hospital,

Sdr. Boulevard 29,

5000 Odense C, Denmark

e-mail: jakob.grauslund@ouh.regionsyddanmark.dk

L. Hodgson $\cdot$ R. Kawasaki $\cdot$ T. Y. Wong

Centre for Eye Research Australia,

Royal Victorian Eye and Ear Hospital, University of Melbourne,

Melbourne, VIC, Australia

\author{
A. Green \\ Center for National Clinical Databases, South, \\ Odense University Hospital, \\ Odense, Denmark \\ T. Y. Wong \\ Singapore Eye Research Institute, Yong Loo Lin School of Medicine, \\ National University of Singapore, \\ Singapore, Singapore
}

Results Retinal photographs were gradable for 188 of 208 patients $(90.3 \%)$. The median age and duration of diabetes for patients with gradable photos were 57.9 and 42 years, respectively. After multivariate adjustments, individuals with narrower retinal arterioles were more likely to have nephropathy (OR 2.17, 95\% CI 1.29-3.68, per SD decrease in CRAE) and macrovascular disease (OR 3.17, 95\% CI 1.59-6.34, per SD decrease in CRAE), but not neuropathy (OR 1.10, 95\% CI 0.70-1.71, per SD decrease in CRAE). Retinal venular calibre was not associated with any microor macrovascular complications.

Conclusions/interpretation In type 1 diabetic patients, retinal arteriolar narrowing is associated with nephropathy and macrovascular disease independently of other clinical characteristics. If supported by further prospective studies, measurement of retinal vessel diameter may allow a non-invasive evaluation of the risk of diabetes-related complications.

Keywords Macrovascular disease - Nephropathy

Neuropathy $\cdot$ Retinal vessel calibre $\cdot$ Type 1 diabetes

\author{
Abbreviations \\ AVR Arteriolar venular ratio \\ CRAE Central retinal artery equivalent \\ CRVE Central retinal vein equivalent \\ WESDR Wisconsin Epidemiologic Study of Diabetic \\ Retinopathy
}

\section{Introduction}

Examination of the human retinal vasculature allows direct in vivo observation of the microvascular system. Quantitative assessments of retinal vascular calibre have shown 
associations with the risk of micro- $[1,2]$ and macrovascular [1, 3-5] disease in studies of type 1 diabetic patients. Further research is important to determine the value of retinal imaging as a non-invasive test to predict micro- and macrovascular complications in patients with diabetes. However, most studies on retinal calibre have been carried out in the general population and in individuals with type 2 diabetes. The aim of this study was to examine the relationship between retinal vascular calibre and long-term micro- and macrovascular complications in a population-based cohort of Danish type 1 diabetic patients.

\section{Methods}

Study population Study participants were identified based on insulin prescriptions in a population-based cohort of all type 1 diabetic patients from Fyn County, Denmark, with an onset of diabetes before the age of $30(n=727)$ identified as of 1 July 1973. In 2007, 208 of 320 surviving patients $(65.0 \%)$ agreed to participate in an examination.

Examination The examinations took place between 1 March 2007 and 1 March 2008 and were performed by a single examiner (J. Grauslund). The study was approved by the local ethics committee, and all participants gave a written informed consent. A full medical history was obtained. An Omron M4 (Omron, Matsusaka, Japan) was used for blood pressure measurement. A history of smoking was obtained. One pack-year was defined as 20 cigarettes smoked in 1 year.

Measurement of $\mathrm{HbA}_{1 \mathrm{c}}$ was performed by cation exchange chromatography using Tosoh G8 HPLC equipment (Medinor, Broendby, Denmark) with reagents as recommended by the supplier. Nephropathy was evaluated in a single spot urine sample. Definitions were: normoalbuminuria, 0-19 mg/l: microalbuminuria, 20-200 mg/l; and macroalbuminuria, $201 \mathrm{mg} / 1$ and above. A monofilament test (Bailey Instruments, Manchester, UK) was used to test for neuropathy. Data on macrovascular disease were obtained from the Danish National Patient Registry. Macrovascular disease was defined as a history of at least one of the following events: stroke, myocardial infarction, angina pectoris, percutaneous coronary intervention, coronary bypass surgery or limb amputation.

Retinal photography and assessment Pupils were dilated and fundus photographs were taken in both eyes. Nine $45^{\circ}$ colour fields were captured with a Topcon TRC-NW6S (Topcon, Tokyo, Japan) and were automatically combined as a mosaic image with IMAGEnet. Photographs were graded for proliferative diabetic retinopathy at the Ocular Epidemiology Research Services, Madison, WI, USA. For each patient, the final category was determined by the worse eye.

Retinal vascular calibre was measured using a computerbased program following previously validated protocols [6]. One disc-centred image was used for this measurement. For each photograph, the largest six arterioles and venules coursing through an area one-half to one disc diameter from the optic disc were measured and summarised as the central retinal artery equivalent (CRAE) and the central retinal vein equivalent (CRVE) using formulae proposed by Knudtson et al. [6]. The arteriolar venular ratio (AVR) was defined as the ratio of CRAE to CRVE. The AVR has been suggested as a useful indicator for changes in retinal arteriolar calibre. Use of the AVR as a common measure of retinal vascular calibres also minimises the effect of magnification differences associated with refractive error [7].

A high inter-eye correlation has been showed between right and left eyes, and consequently we only used the right eye for grading [8]. Left eye measurements were used when photographs of the right eye were not of sufficient quality for grading. All measurements were performed by a single trained grader (J. Grauslund). Intergrader reproducibility, determined by re-grading randomly chosen photographs from 20 individuals, was high (intra-class correlation coefficients of 0.96 and 0.99 for CRAE and CRVE, respectively).

Statistical analyses Categorical data are presented as percentages and continuous data are presented as medians and interquartile ranges (25th to 75 th percentiles). All statistical differences were tested at the 0.05 level. The $\chi^{2}$ test was used for categorical data. For continuous data we used the Mann-Whitney test for two groups and Cuzick's test for trend for several groups. Multiple logistic regression models were used to obtain the ORs for nephropathy, neuropathy and macrovascular disease in relation to retinal calibre. Two models were used: an age- and sex-adjusted model and a multivariate model. The latter was adjusted for age, sex, duration of diabetes, $\mathrm{HbA}_{1 \mathrm{c}}$, systolic blood pressure and pack-years of smoking. Furthermore, CRAE and CRVE were entered simultaneously to control for errors due to magnification. Stata Intercooled 9.2 was used for all analyses.

\section{Results}

Two hundred and eight patients were examined. Twenty patients had to be excluded as retinal vessel calibre could not be measured in any eye. The median age and duration of diabetes for patients included were 57.9 and 42 years, respectively. 
In univariate analyses, narrow retinal arteriolar calibre was related to poor glycaemic control and systolic blood pressure (Table 1). Venular narrowing was also related to systolic blood pressure and, furthermore, a larger CRVE was found among smokers. Finally, a low AVR was associated with a short duration of diabetes and smoking.

In additional univariate analyses, arteriolar narrowing was related to nephropathy and proliferative diabetic retinopathy. Median CRAEs were $143.3 \mu \mathrm{m}, 134.0 \mu \mathrm{m}$ and $127.1 \mu \mathrm{m}$ for patients with normo-, micro- and macroalbuminuria, respectively $(p<0.01)$, and vessel calibre was significantly narrower in patients who had panretinal photocoagulation for proliferative retinopathy (CRAE: 131.1 vs $147.5 \mu \mathrm{m}, p<0.01$; CRVE: 198.5 vs $214.9 \mu \mathrm{m}, p<0.01$; AVR 0.65 vs $0.68, p=$ 0.02) (data not shown).

A logistic regression was performed to evaluate the correlation between retinal vessel calibre and nephropathy, neuropathy and macrovascular diseases. Results are pre- sented in Table 2 for the age- and sex-adjusted model and the multivariate model. In multivariate analyses, associations were found for retinal arteriolar calibre. The odds ratios for nephropathy and macrovascular disease were 2.17 (95\% CI 1.29-3.68) and 3.17 (95\% CI 1.59-6.34), respectively, for each SD decrease in CRAE. The same associations were found for AVR. However, there were no associations between CRVE and any of the complications.

\section{Discussion}

In this study, we report on associations between retinal vascular calibre, measured from fundus photographs using a computer-based program, and diabetes-related micro- and macrovascular complications in a population-based cohort of Danish type 1 diabetic patients. We found that retinal arteriolar narrowing (and a low AVR) was associated with

Table 1 Relationship between retinal vessel calibre and clinical characteristics

\begin{tabular}{|c|c|c|c|c|c|c|c|}
\hline \multirow[t]{2}{*}{ Clinical characteristic } & \multirow[t]{2}{*}{$n$} & \multicolumn{2}{|c|}{$\begin{array}{l}\text { Retinal arteriolar calibre } \\
\text { (CRAE in } \mu \mathrm{m})\end{array}$} & \multicolumn{2}{|c|}{$\begin{array}{l}\text { Retinal venular calibre } \\
\text { (CRVE in } \mu \mathrm{m})\end{array}$} & \multicolumn{2}{|l|}{ AVR } \\
\hline & & Median (IQR) & $p$ value & Median (IQR) & $p$ value & Median (IQR) & $p$ value \\
\hline Age (years) & & & 0.32 & & 0.10 & & 0.07 \\
\hline $37.7-52.7$ & 62 & $141.5 \pm 24.9$ & & $208.9 \pm 36.1$ & & $0.65 \pm 0.09$ & \\
\hline $53.0-61.8$ & 62 & $142.9 \pm 24.4$ & & $211.1 \pm 29.8$ & & $0.67 \pm 0.08$ & \\
\hline $61.9-84.4$ & 64 & $139.0 \pm 17.6$ & & $208.0 \pm 37.9$ & & $0.69 \pm 0.10$ & \\
\hline Duration of diabetes (years) & & & 0.42 & & 0.06 & & $0.04 *$ \\
\hline $34-39$ & 64 & $142.9 \pm 20.4$ & & $215.8 \pm 33.4$ & & $0.65 \pm 0.08$ & \\
\hline $40-45$ & 58 & $140.0 \pm 24.8$ & & $205.5 \pm 36.8$ & & $0.68 \pm 0.11$ & \\
\hline $46-70$ & 66 & $139.8 \pm 21.8$ & & $209.3 \pm 35.2$ & & $0.68 \pm 0.09$ & \\
\hline $\mathrm{HbA}_{1 \mathrm{c}}(\%)$ & & & $0.01 *$ & & 0.22 & & 0.24 \\
\hline $5.4-7.2$ & 63 & $142.6 \pm 22.7$ & & $209.5 \pm 25.0$ & & $0.68 \pm 0.08$ & \\
\hline $7.3-8.0$ & 60 & $142.0 \pm 23.6$ & & $209.3 \pm 29.5$ & & $0.66 \pm 0.08$ & \\
\hline $8.1-13.3$ & 62 & $133.8 \pm 23.3$ & & $207.3 \pm 48.9$ & & $0.67 \pm 0.09$ & \\
\hline Systolic BP (mmHg) & & & $0.02 *$ & & $0.04 *$ & & 0.83 \\
\hline $102-139$ & 64 & $145.1 \pm 25.0$ & & $211.9 \pm 41.3$ & & $0.68 \pm 0.11$ & \\
\hline $140-155$ & 61 & $140.5 \pm 19.2$ & & $209.5 \pm 23.6$ & & $0.67 \pm 0.07$ & \\
\hline $156-235$ & 63 & $136.0 \pm 22.0$ & & $207.3 \pm 39.4$ & & $0.66 \pm 0.09$ & \\
\hline Diastolic BP (mmHg) & & & 0.60 & & 0.17 & & 0.13 \\
\hline $52-71$ & 62 & $141.2 \pm 24.3$ & & $212.9 \pm 32.1$ & & $0.66 \pm 0.09$ & \\
\hline $72-79$ & 64 & $140.7 \pm 24.8$ & & $206.8 \pm 42.0$ & & $0.69 \pm 0.07$ & \\
\hline $80-109$ & 62 & $140.6 \pm 20.3$ & & $207.6 \pm 26.2$ & & $0.67 \pm 0.09$ & \\
\hline Smoking (pack-years) & & & 0.44 & & $0.01^{*}$ & & $0.03 *$ \\
\hline 0 & 78 & $140.0 \pm 29.4$ & & $204.8 \pm 30.8$ & & $0.68 \pm 0.09$ & \\
\hline $1-20$ & 54 & $141.5 \pm 17.4$ & & $214.0 \pm 31.8$ & & $0.68 \pm 0.08$ & \\
\hline $21-120$ & 56 & $142.1 \pm 22.5$ & & $214.9 \pm 41.7$ & & $0.64 \pm 0.09$ & \\
\hline
\end{tabular}

Data are presented as medians and interquartile ranges (25th to 75 th percentiles), and $p$ was tested for trend

*Statistically significant, $p<0.05$ 
Table 2 Association of retinal vascular calibre and diabetes-related complications

\begin{tabular}{|c|c|c|c|c|c|c|c|}
\hline \multirow{2}{*}{$\begin{array}{l}\text { Retinal vascular } \\
\text { calibre }\end{array}$} & \multirow[t]{2}{*}{$n$} & \multicolumn{2}{|l|}{ Nephropathy } & \multicolumn{2}{|l|}{ Neuropathy } & \multicolumn{2}{|c|}{ Macrovascular disease } \\
\hline & & $\begin{array}{l}\text { Age- and } \\
\text { sex-adjusted }\end{array}$ & Multivariate & $\begin{array}{l}\text { Age- and } \\
\text { sex-adjusted }\end{array}$ & Multivariate & $\begin{array}{l}\text { Age- and } \\
\text { sex-adjusted }\end{array}$ & Multivariate \\
\hline \multicolumn{8}{|l|}{ CRAE $(\mu \mathrm{m})$} \\
\hline $\begin{array}{l}\text { Per SD decrease, } \\
17.4 \mu \mathrm{m}\end{array}$ & & $1.68(1.20-2.34)^{*}$ & $2.17(1.29-3.68)^{*}$ & $1.27(0.94-1.72)$ & $1.10(0.70-1.71)$ & $1.95(1.34-2.86)^{*}$ & $3.17(1.59-6.34)^{*}$ \\
\hline Tertile $1, \leq 132.4 \mu \mathrm{m}$ & 62 & $2.28(1.04-5.00)^{*}$ & $2.28(0.80-6.55)$ & $1.41(0.69-2.89)$ & $0.93(0.37-2.38)$ & $3.99(1.61-9.93)^{*}$ & $6.86(1.75-26.9)^{*}$ \\
\hline $\begin{array}{l}\text { Tertile } 2,132.5- \\
147.3 \mu \mathrm{m}\end{array}$ & 62 & $1.28(0.56-2.91)$ & $1.34(0.53-3.38)$ & $0.62(0.30-1.30)$ & $0.51(0.23-1.14)$ & $0.69(0.22-2.13)$ & $0.51(0.12-2.12)$ \\
\hline Tertile $3, \geq 147.4 \mu \mathrm{m}$ & 62 & 1.00 & 1.00 & 1.00 & 1.00 & 1.00 & 1.00 \\
\hline \multicolumn{8}{|l|}{ CRVE $(\mu \mathrm{m})$} \\
\hline $\begin{array}{l}\text { Per SD increase, } \\
29.4 \mu \mathrm{m}\end{array}$ & & $0.90(0.65-1.23)$ & $1.43(0.85-2.40)$ & $0.80(0.59-1.09)$ & $0.84(0.53-1.33)$ & $0.86(0.60-1.25)$ & $1.78(0.91-3.46)$ \\
\hline Tertile $1, \leq 199.5 \mu \mathrm{m}$ & 62 & 1.00 & 1.00 & 1.00 & 1.00 & 1.00 & 1.00 \\
\hline $\begin{array}{l}\text { Tertile } 2,199.6- \\
218.9 \mu \mathrm{m}\end{array}$ & 62 & $0.60(0.28-1.29)$ & $0.97(0.40-2.37)$ & $0.56(0.27-1.15)$ & $0.75(0.33-1.67)$ & $0.51(0.20-1.27)$ & $1.16(0.36-3.76)$ \\
\hline Tertile $3, \geq 219.0 \mu \mathrm{m}$ & 62 & $0.65(0.30-1.40)$ & $1.17(0.40-3.44)$ & $0.78(0.38-1.59)$ & $1.16(0.44-3.06)$ & $0.83(0.35-1.96)$ & $2.69(0.67-10.7)$ \\
\hline \multicolumn{8}{|l|}{ AVR } \\
\hline Per SD decrease, 0.07 & & $1.69(1.17-2.44)^{*}$ & $1.48(1.01-2.16)^{*}$ & $1.00(0.74-1.36)$ & $0.95(0.69-1.31)$ & $2.08(1.32-3.28)^{*}$ & $1.79(1.09-2.95)^{*}$ \\
\hline Tertile $1, \leq 0.63$ & 56 & $3.17(1.44-6.95)^{*}$ & $2.34(1.01-5.39)^{*}$ & $0.92(0.44-1.91)$ & $0.80(0.37-1.73)$ & $3.71(1.40-9.82)^{*}$ & $2.54(0.85-7.59)$ \\
\hline Tertile $2,0.64-0.69$ & 60 & $1.04(0.46-2.37)$ & $0.88(0.37-2.07)$ & $1.05(0.52-2.12)$ & $0.94(0.46-1.93)$ & $2.45(0.93-6.44)$ & $2.24(0.78-6.43)$ \\
\hline Tertile $3, \geq 0.70$ & 70 & 1.00 & 1.00 & 1.00 & 1.00 & 1.00 & 1.00 \\
\hline
\end{tabular}

Values are odds ratio $(95 \% \mathrm{CI})$

The multivariate models were adjusted for age, sex, duration of diabetes, $\mathrm{HbA}_{1 \mathrm{c}}$, systolic blood pressure and pack-years of smoking. Multivariate models for subgroups stratified according to CRAE, CRVE or AVR were also adjusted for the stratifying variable. * Statistically significant, $p<0.05$

nephropathy and macrovascular disease, independently of other clinical characteristics. No relationships were found with retinal venular diameter.

The association of retinal arteriolar narrowing and proteinuria confirms cross-sectional results from the Wisconsin Epidemiologic Study of Diabetic Retinopathy (WESDR) [1]. Patients with type 1 diabetes with proteinuria had narrower retinal arterioles - by about $20 \mu \mathrm{m}$ - than those without proteinuria. However, the same association was not found in a 16 year follow-up of the WESDR [2]. Wong and coauthors speculate that selective mortality in individuals with retinal arteriolar narrowing and renal disease might be the reason for the lack of associations [2].

In multivariate analyses, we found macrovascular disease to be associated with narrower retinal arterioles. The same association was also seen in the WESDR study for type 1 diabetic patients $[1,4]$. The association of retinal arteriolar disease with macrovascular disease may be caused by microvascular changes in the heart and brain similar to those found in the retina.

We did not find an association between retinal vascular calibre and neuropathy. We have no knowledge of other studies in type 1 diabetes in which microvascular risk factors for neuropathy and nephropathy were directly compared. Our findings indicate that there may be subtle differences in the pathogenesis of neuropathy as compared with nephropathy. This requires further investigation.

We observed a generalised narrowing of retinal arterioles and venules in eyes that had been treated with panretinal photocoagulation. This is consistent with clinical observation of narrower retinal vessels after laser treatment [1], reflecting reduced blood flow secondary to a lower metabolic demand caused by the destruction of viable retinal tissue [9].

A major strength of the present study is the populationbased design that minimises the risk of referral bias. Some limitations need to be addressed. First, these observations were cross-sectional, which limits our ability to determine antecedent-consequent relations. Thus, we cannot determine if retinal arteriolar narrowing predicts nephropathy and macrovascular disease or vice versa. Second, the observations reported may have been affected by survival bias given the well-known association between retinal arteriolar narrowing and mortality in diabetic patients [4]. Finally, we were not able to control for variations in pulsatility associated with the cardiac cycle. A variation in diameter of $6-17 \%$ for arterioles and $2-11 \%$ for venules has been reported [10]. Our findings may be attenuated by this variation.

In summary, in a population-based cohort of Danish type 1 diabetic patients, we demonstrate associations between 
retinal arteriolar narrowing, nephropathy and macrovascular disease, independent of common risk factors. If supported by prospective studies, retinal vascular measurements may be useful as an additional tool to predict diabetic patients at risk of debilitating complications.

Acknowledgements This work was supported by grants from the Velux Foundation, the Danish Eye Health Society, the Sehested Hansen's Foundation, the Danish Diabetes Association, the Synoptik Foundation, the A.P. Møller Foundation for the Advancement of Medical Science, the Danish Society of Ophthalmology and the Institute of Clinical Research at University of Southern Denmark. Additional support was provided by a Juvenile Diabetes Research Foundation Innovation Grant.

Duality of interest The authors declare that there is no duality of interest associated with this manuscript.

\section{References}

1. Klein R, Klein BE, Moss SE et al (2003) Retinal vascular abnormalities in persons with type 1 diabetes: the Wisconsin Epidemiologic Study of Diabetic Retinopathy: XVIII. Ophthalmology 110:2118-2125

2. Wong TY, Shankar A, Klein R, Klein BE (2004) Retinal vessel diameters and the incidence of gross proteinuria and renal insufficiency in people with type 1 diabetes. Diabetes 53: 179-184

3. Miller RG, Prince CT, Klein R, Orchard TJ (2009) Retinal vessel diameter and the incidence of coronary artery disease in type 1 diabetes. Am J Ophthalmol 147:653-660

4. Klein BE, Klein R, McBride PE et al (2004) Cardiovascular disease, mortality, and retinal microvascular characteristics in type 1 diabetes: Wisconsin Epidemiologic Study of Diabetic Retinopathy. Arch Intern Med 164:1917-1924

5. Moss SE, Klein R, Klein BE, Wong TY (2003) Retinal vascular changes and 20-year incidence of lower extremity amputations in a cohort with diabetes. Arch Intern Med 163:2505-2510

6. Knudtson MD, Lee KE, Hubbard LD, Wong TY, Klein R, Klein BE (2003) Revised formulas for summarizing retinal vessel diameters. Curr Eye Res 27:143-149

7. Wong TY, Wang JJ, Rochtchina E, Klein R, Mitchell P (2004) Does refractive error influence the association of blood pressure and retinal vessel diameters? The Blue Mountains Eye Study. Am J Ophthalmol 137:1050-1055

8. Leung H, Wang JJ, Rochtchina E et al (2003) Computer-assisted retinal vessel measurement in an older population: correlation between right and left eyes. Clin Experiment Ophthalmol 31:326330

9. Grunwald JE, Riva CE, Brucker AJ, Sinclair SH, Petrig BL (1986) Effect of panretinal photocoagulation on retinal blood flow in proliferative diabetic retinopathy. Ophthalmology 93: 590-595

10. Knudtson MD, Klein BE, Klein R et al (2004) Variation associated with measurement of retinal vessel diameters at different points in the pulse cycle. Br J Ophthalmol 88:57-61 